\title{
Parkinson's disease patients' short chain fatty acids production capacity after in vitro fecal fiber fermentation
}

\author{
Florence Baert $\mathbb{D}^{1,2}$, Christophe Matthys $\mathbb{D}^{2,3}$, Jarissa Maselyne ${ }^{1}$, Christof Van Poucke ${ }^{1}$, Els Van Coillie ${ }^{1}$, Bruno Bergmans ${ }^{4,5}$ and \\ Geertrui Vlaemynck (iD ${ }^{1 凶}$
}

Animal models indicate that butyrate might reduce motor symptoms in Parkinson's disease. Some dietary fibers are butyrogenic, but in Parkinson's disease patients their butyrate stimulating capacity is unknown. Therefore, we investigated different fiber supplements' effects on short-chain fatty acid production, along with potential underlying mechanisms, in Parkinson's patients and age-matched healthy controls. Finally, it was investigated if this butyrate production could be confirmed by using fiber-rich vegetables. Different fibers $(n=40)$ were evaluated by in vitro fermentation experiments with fecal samples of Parkinson's patients $(n=24)$ and age-matched healthy volunteers $(n=39)$. Short-chain fatty acid production was analyzed by headspace solid-phase micro-extraction gas chromatography-mass spectrometry. Clostridium coccoides and C. leptum were quantified through 16S-rRNA gene-targeted group-specific qPCR. Factors influencing short-chain fatty acid production were investigated using linear mixed models. After fiber fermentation, butyrate concentration varied between $25.6 \pm 16.5 \mu \mathrm{mol} / \mathrm{g}$ and $203.8 \pm 91.9 \mu \mathrm{mol} / \mathrm{g}$ for Parkinson's patients and between $52.7 \pm 13.0 \mu \mathrm{mol} / \mathrm{g}$ and $229.5 \pm 42.8 \mu \mathrm{mol} / \mathrm{g}$ for controls. Inulin had the largest effect, while xanthan gum had the lowest production. Similar to fiber supplements, inulin-rich vegetables, but also fungal $\beta$-glucans, stimulated butyrate production most of all vegetable fibers. Parkinson's disease diagnosis limited short-chain fatty acid production and was negatively associated with butyrate producers. Butyrate kinetics during $48 \mathrm{~h}$ fermentation demonstrated a time lag effect in Parkinson's patients, especially in fructo-oligosaccharide fermentation. Butyrate production can be stimulated in Parkinson's patients, however, remains reduced compared to healthy controls. This is a first step in investigating dietary fiber's potential to increase short-chain fatty acids in Parkinson's disease.

npj Parkinson's Disease (2021)7:72; https://doi.org/10.1038/s41531-021-00215-5

\section{INTRODUCTION}

As reviewed by Elfil et al. (2020), changes in the gut microbiome composition are thought to play a role in the pathophysiology of Parkinson's disease (PD) and could be a potential target in future therapies ${ }^{1}$. The gut microbiome composition of PD patients is characterized by a lower number of butyrate-producing bacteria and a more pro-inflammatory profile $\mathrm{e}^{2-7}$, combined with lower fecal short-chain fatty acids (SCFA) concentrations ${ }^{5}$. SCFA are hypothesized to be important gut-brain axis mediators ${ }^{8}$. In vitro studies have demonstrated that SCFA cross the blood-brain barrier, moreover low concentrations of butyrate and propionate have been reported in healthy volunteers' brains ${ }^{9,10}$. PD animal models demonstrated that butyrate administration improves motor deficits, reduces inflammation and dopamine deficiency ${ }^{11-13}$. Matt et al. (2018) showed that both intra-peritoneal butyrate administration and a high dietary fiber diet resulted in reduced expression of pro-inflammatory genes in aged mice's brains $^{14}$. In contrast, results by Sampson et al. (2016) demonstrated that administration of an SCFA mixture promoted neuroinflammation in a germ-free PD mouse model ${ }^{15}$. However, the effect of the SCFA may depend on the concentration and on the composition of the SCFA mixture ${ }^{16}$. The administered concentration of the SCFA mixture may not resemble the concentration of microbial produced SCFA ${ }^{16}$. In fact, beneficial effects of administration of a low dose of butyrate in a mouse model of autism have been reported, whereas a high dose did not exert any effects ${ }^{17}$. Except for the study by Sampson et al. (2016), the above-mentioned animal studies suggest that SCFA, particularly butyrate, are noteworthy to investigate further. The hypothesis is that enhancing colonic SCFA production would be beneficial in PD.

Colonic SCFA concentrations can be increased by administering butyrate as such (both oral and rectal) and/or by increasing the butyrate-producing bacteria through fecal transplantation, probiotics, or dietary fiber ${ }^{18-25}$. Disadvantages of enema's, fecal transplantation, or probiotics compared to fiber may be the patient's discomfort and/or hesitance towards their use $\mathrm{e}^{26}$. Furthermore, manufacturing and digestion can decrease probiotics' viability, thereby limiting their effects ${ }^{27}$. Hence, dietary fiber consumption could be an acceptable option. Especially as PD patients have a higher daily fiber intake compared to healthy controls $(\mathrm{HC})^{28-30}$.

Following the suggestion of Elfil et al. (2020) of SCFA modification as a potential therapeutic strategy ${ }^{1}$, we hypothesize that a high fiber diet can increase butyrate production in PD patients through colonic fiber fermentation. Although a first step is to evaluate the SCFA producing capacity of PD patients' gut microbiota.

Therefore, we aim to increase SCFA production in fecal samples of PD patients through in vitro fermentation experiments with different types of fiber supplements, compared to SCFA

${ }^{1}$ Department Technology and Food, Flanders Research Institute for Agriculture, Fisheries and Food, Melle, Belgium. ${ }^{2}$ Clinical and Experimental Endocrinology, Department of Chronic Diseases and Metabolism, KU Leuven, O\&N I, Leuven, Belgium. ${ }^{3}$ Department of Endocrinology, University Hospitals Leuven, Campus Gasthuisberg, Leuven, Belgium. ${ }^{4}$ Department of Neurology, AZ Sint-Jan Brugge-Oostende AV, Bruges, Belgium. ${ }^{5}$ Department of Neurology, University Hospitals Ghent, Ghent, Belgium.

出mail: geertrui.vlaemynck@ilvo.vlaanderen.be 
production in fecal samples of age-matched HC. By investigating fibers' effects on butyrate-producing bacteria and SCFA production kinetics, we aim to increase understanding of mechanisms behind colonic SCFA production in PD patients. Finally, we evaluate whether vegetable and quinoa fiber can confirm the in vitro SCFA production results obtained by fiber supplements.

\section{RESULTS}

In total, 63 participants were included, 24 PD patients and $39 \mathrm{HC}$. A minimum of 4 fecal samples of both PD patients and HC were used per fiber substrate, see also Supplementary Table 1.

The mean Hoehn and Yahr score of PD patients was $2.3 \pm 0.5$, ranging from 1.5-3. An overview of all participants' characteristics is shown in Table 1. A significant difference in the ratio of men to women $(p=0.00007)$, BMl $(p=0.01)$, reported weight loss $(p=$ $0.02)$, and antidepressant intake $(p=0.006)$ was found between $\mathrm{PD}$ patients and $\mathrm{HC}$.

\section{Fiber supplements}

Univariate analyses of baseline SCFA concentrations prior to fermentation (Blank T0) demonstrated that PD patients had significantly lower concentrations of acetate $(p=0.002)$ and total SCFA $(p=0.008)$ compared to HC, for butyrate a trend $(p=0.09)$ towards a lower concentration was found. Univariate analyses demonstrated that PD patients had overall significantly reduced acetate $(p=0.03)$ and butyrate $(p=0.01)$ production compared to HC, for total SCFA a trend $(p=0.09)$ towards lower production was found. Fiber supplements, except for xanthan gum, all stimulated butyrate production in PD and HC (see Fig. 1), however large interindividual variability in SCFA production was found (see Supplementary Figs. 1-7). An overview of univariate analyses in all participants is given in Supplementary Table 2. PD medication, disease duration, and antidepressant intake's influence on SCFA production was analyzed within PD patients. Levodopa was negatively associated with isobutyrate production $(p=0.04)$ and a trend towards lower isovalerate production $(p=0.05)$ was found. Catechol-O-methyltransferase (COMT) inhibitor was positively associated with total SCFA production $(p=0.04)$. Disease duration was positively associated with valerate production $(p=0.002)$.

Multivariate analysis demonstrated that fiber type and PD diagnosis were the strongest predictors of SCFA production, compared to other fixed factors. Fiber stimulated acetate, butyrate, and total SCFA production significantly, whereas PD diagnosis significantly limited their overall production. The final multivariate models are shown in Table 2A. Of all fiber types, inulins stimulated butyrate production most $(p<0.0001)$, see Figs 1 and 2 . Oligosaccharides $(p<0.0001)$ increased butyrate production more compared to RS, pectins, and fibers consisting of hemicelluloses, cellulose, and lignin, see Fig. 2. Mean butyrate production increase in $\mathrm{PD}$ and $\mathrm{HC}$ per fiber type is shown in Supplementary Table 3 and Supplementary Fig. 8.

Visual inspection of Fig. 1 suggested PD patients had a higher mean valerate production after fermentation with fiber supplements than HC. In $21 \%$ of PD patients, valerate increased between 1.3 and 27 times the valerate concentration in blanks after fermentation with the majority of fiber supplements tested in those PD patients' samples. However, multivariate analysis showed no effect of PD diagnosis on valerate production.

The kinetic profile indicated that 3-12 $\mathrm{h}$ after inulin, RS, and FOS fermentation, PD had lower butyrate production compared to $\mathrm{HC}$, see Fig. 3 and Table $2 \mathrm{C}^{1-7}$. The post-hoc analysis demonstrated only a trend towards a difference between PD and HC in butyrate production after $3 \mathrm{~h}$ FOS fermentation $(p=0.06)$. The area under the curve (AUC) after fermentation with all 3 fibers was higher in $\mathrm{HC}$ compared to PD, although the difference was not statistically significant (Supplementary Table 4). No significant differences in
Table 1. Overview of participant's characteristics.

\begin{tabular}{|c|c|c|c|}
\hline & $\begin{array}{l}\text { PD } \\
\text { patients } \\
(n=24)\end{array}$ & $\begin{array}{l}\text { Healthy } \\
\text { volunteers } \\
(n=39)\end{array}$ & $P$-value \\
\hline \multicolumn{4}{|l|}{ Sex } \\
\hline Men/women & $21 / 3$ & $14 / 25$ & 0.00007 \\
\hline \multicolumn{4}{|l|}{ Age } \\
\hline Age (years), mean $\pm S D$ & $62.5 \pm 6.9$ & $60.5 \pm 4.4$ & 0.13 \\
\hline Duration of disease (years), mean $\pm S D$ & $6.1 \pm 5.0$ & - & - \\
\hline $\begin{array}{l}\text { Duration of disease (years), } \\
\text { minimum-maximum }\end{array}$ & $1-18$ & - & - \\
\hline BMI $\left(\mathrm{kg} / \mathrm{m}^{2}\right)$, mean $\pm \mathrm{SD}$ & $24.2 \pm 2.0$ & $22.9 \pm 1.9$ & 0.01 \\
\hline Dietary fiber intake (g/day), mean $\pm S D$ & $22.9 \pm 9.7$ & $24.5 \pm 10.5$ & 0.54 \\
\hline Bristol Stool Form Scale & & & 0.11 \\
\hline Type 1 (\%) & 8.3 & 2.6 & \\
\hline Type 2 (\%) & 12.5 & 10.3 & \\
\hline Type 3 (\%) & 37.5 & 12.8 & \\
\hline Type 4 (\%) & 33.3 & 61.5 & \\
\hline Type 5 (\%) & 8.3 & 10.3 & \\
\hline Type 6 (\%) & 0.0 & 2.6 & \\
\hline Type 7 (\%) & 0.0 & 0.0 & \\
\hline $\begin{array}{l}\text { Hoehn and Yahr score, mean } \pm \text { SD }(n= \\
\text { 18) }\end{array}$ & $2.3 \pm 0.5$ & - & - \\
\hline $\begin{array}{l}\text { Hoehn and Yahr score, } \\
\text { minimum-maximum }\end{array}$ & $1.5-3.0$ & - & - \\
\hline Weight loss reported by participants (\%) & 29.2 & 5.7 & 0.02 \\
\hline $\begin{array}{l}\text { Weight loss reported by participants } \\
(\mathrm{kg}) \text {, mean } \pm \text { SD }\end{array}$ & $10.5 \pm 7.9$ & $2.8 \pm 0.4$ & \\
\hline $\begin{array}{l}\text { Weight loss reported by participants } \\
(\mathrm{kg}) \text {, minimum-maximum }\end{array}$ & $3-20$ & $2.5-3$ & \\
\hline \multicolumn{4}{|l|}{ Medication intake } \\
\hline Antidepressants (\%) & 20.8 & 0.0 & 0.006 \\
\hline Medication for high blood pressure (\%) & 20.8 & 15.4 & 0.73 \\
\hline $\begin{array}{l}\text { Medication for high cholesterol } \\
\text { levels (\%) }\end{array}$ & 20.8 & 20.5 & 1.00 \\
\hline Anti-inflammatory drugs (\%) & 4.2 & 5.1 & 1.00 \\
\hline Parkinson's medication (\%) & 91.7 & - & - \\
\hline $\begin{array}{l}\text { Levodopa + DOPA decarboxylase } \\
\text { inhibitor (\%) }\end{array}$ & 75.0 & - & - \\
\hline $\begin{array}{l}\text { Catechol-O-methyltransferase (COMT) } \\
\text { inhibitor (\%) }\end{array}$ & 29.2 & - & - \\
\hline Dopamine agonists (\%) & 45.8 & - & - \\
\hline $\begin{array}{l}\text { Monoamine oxidase-B (MAO B) } \\
\text { inhibitor (\%) }\end{array}$ & 62.5 & - & - \\
\hline Amantadine (\%) & 4.2 & - & - \\
\hline Anticholinergics (\%) & 8.3 & - & - \\
\hline \multicolumn{4}{|c|}{ Predominant Parkinson's disease-related complaints } \\
\hline Movement issues (\%) & 83.3 & - & - \\
\hline Bowel problems (\%) & 20.8 & - & - \\
\hline Negative mood (\%) & 4.2 & - & - \\
\hline Speech difficulties (\%) & 4.2 & - & - \\
\hline Difficulties with eating/drinking (\%) & 8.3 & - & - \\
\hline Hypersalivation (\%) & 8.3 & - & - \\
\hline
\end{tabular}

SD standard deviation, Bristol Stool Form Scale (BSFS) type 1 indicates separate hard to pass fecal lumps, BSFS type 2 indicates lumpy, sausageshaped feces, BSFS type 3 indicates sausage-shaped feces with cracks on the surface, BSFS type 4 indicates smooth, sausage-shaped feces, BSFS type 5 indicates soft fecal blobs with sharp edges (easy to pass), BSFS type 6 indicates mushy soft stools and BSFS type 7 indicates entirely liquid stools. Possible differences in sex, BSFS, and medication intake between PD patients and $\mathrm{HC}$ were analyzed using Fisher's exact test. To evaluate potential differences in BMI, fiber intake, and age between PD and HC, Students T-test and Mann-Whitney U-test were used, depending on normality (evaluated by the Shapiro Wilk test). 

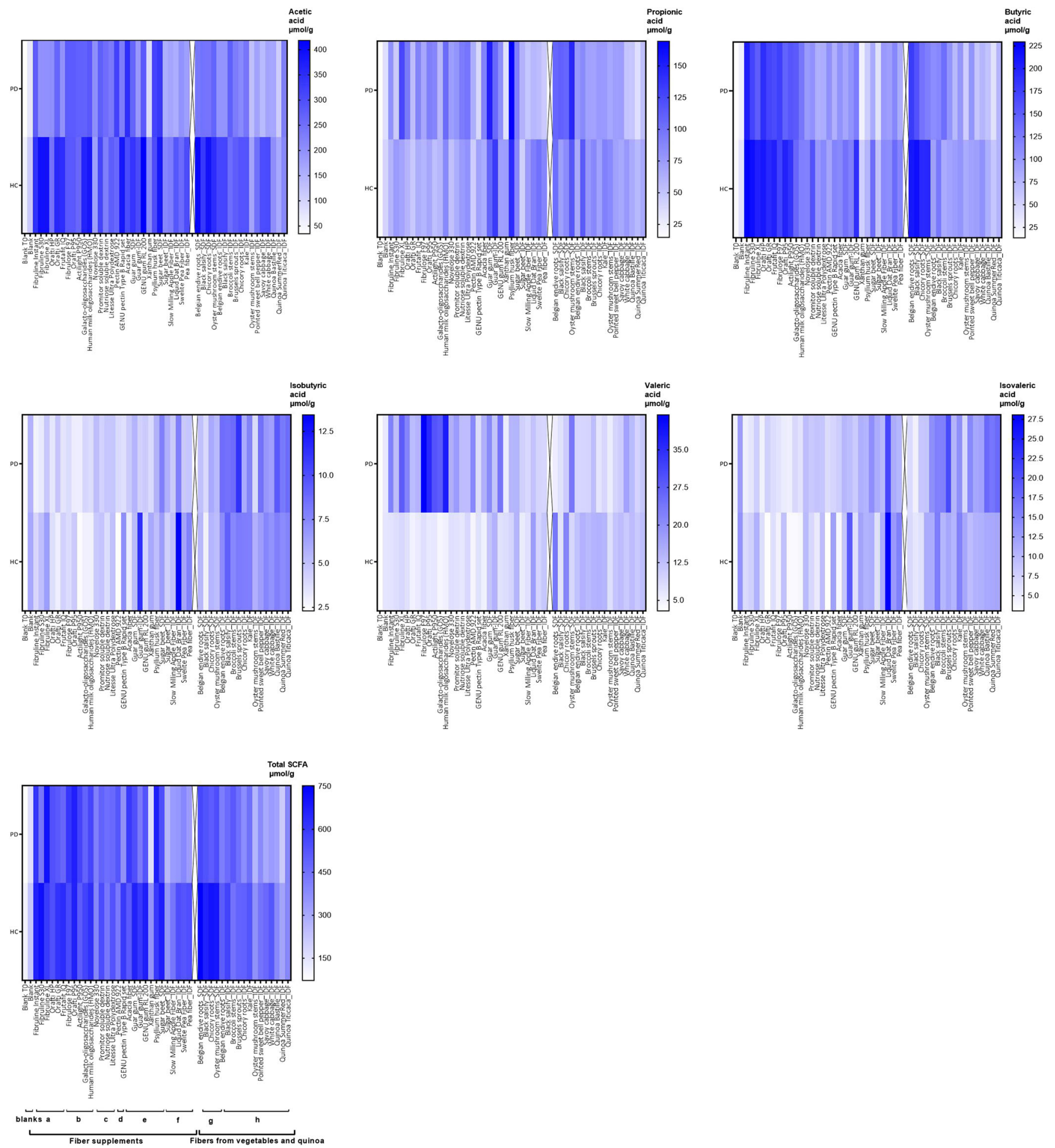

Fig. 1 Overview of mean SCFA production per fiber in Parkinson's Disease patients and healthy controls. a inulins; (b) oligosaccharides; (c) resistant starch/dextrin, polydextrose; (d) pectins; (e) gums; (f) hemicelluloses, cellulose, lignin; (g) vegetable soluble dietary fiber; (h) vegetable/quinoa insoluble dietary fiber; SDF soluble dietary fiber, IDF insoluble dietary fiber, $P D$ Parkinson's patients, $H C$ healthy controls. Potential effects of factors on SCFA production were analyzed using linear mixed models, sex, fiber type, and PD diagnosis were added as fixed factors, the participant was added as a random factor. PD diagnosis resulted in an overall reduction of acetic acid ( $p=0.002)$, butyric acid $(p=0.0001)$, and total SCFA $(p=0.004)$, compared to HC for the fiber supplement experiments (a-f). Similar effects of PD diagnosis on acetic acid $(p=0.01)$, butyric acid $(p=0.097)$, and total SCFA $(p=0.08)$ were observed in the vegetable/quinoa fiber experiments $(\mathbf{g}-\mathbf{h})$. Fibers significantly $(p<2.0 \mathrm{E}-16)$ influenced the production of all SCFA in both experiments. 


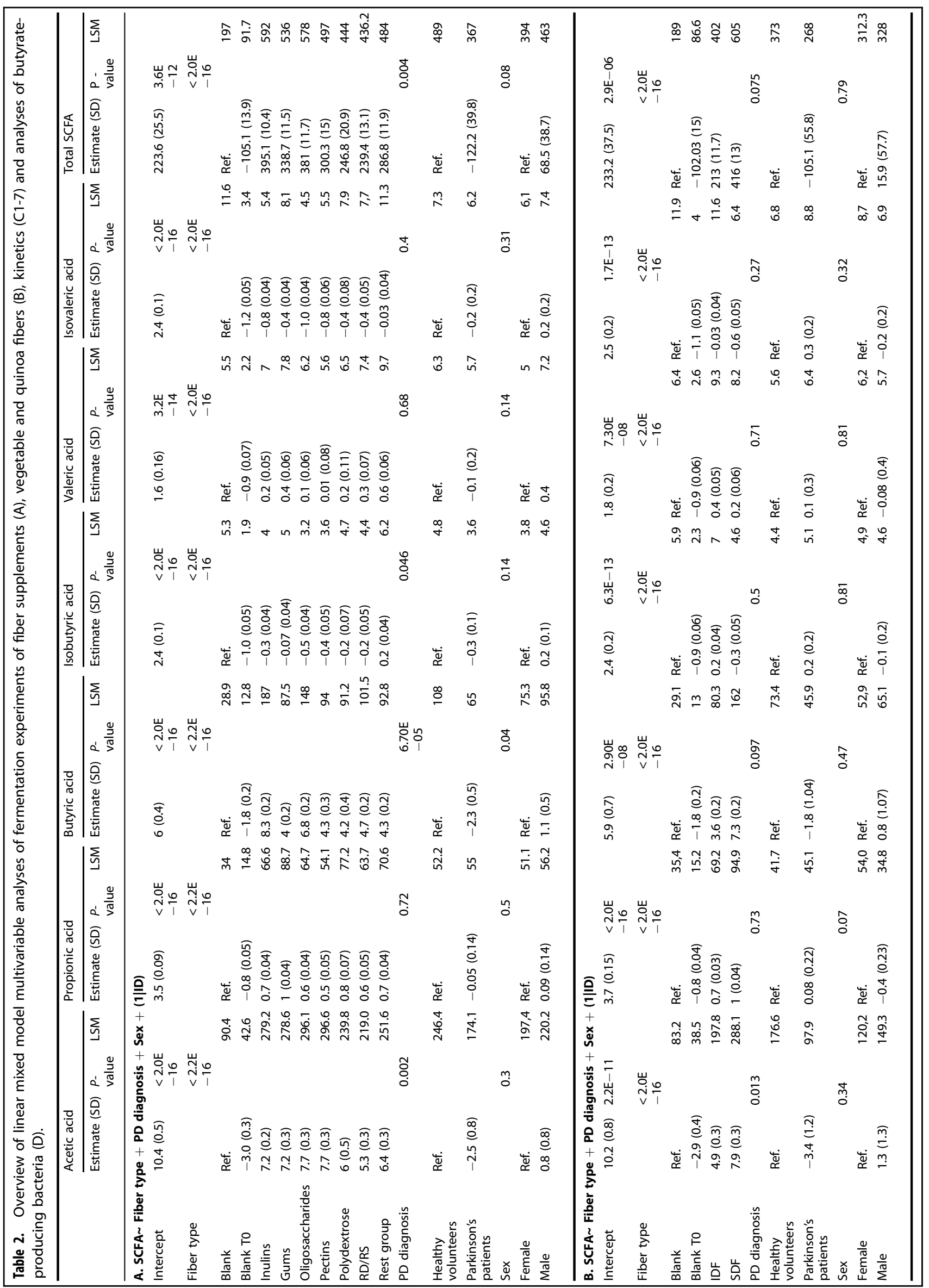




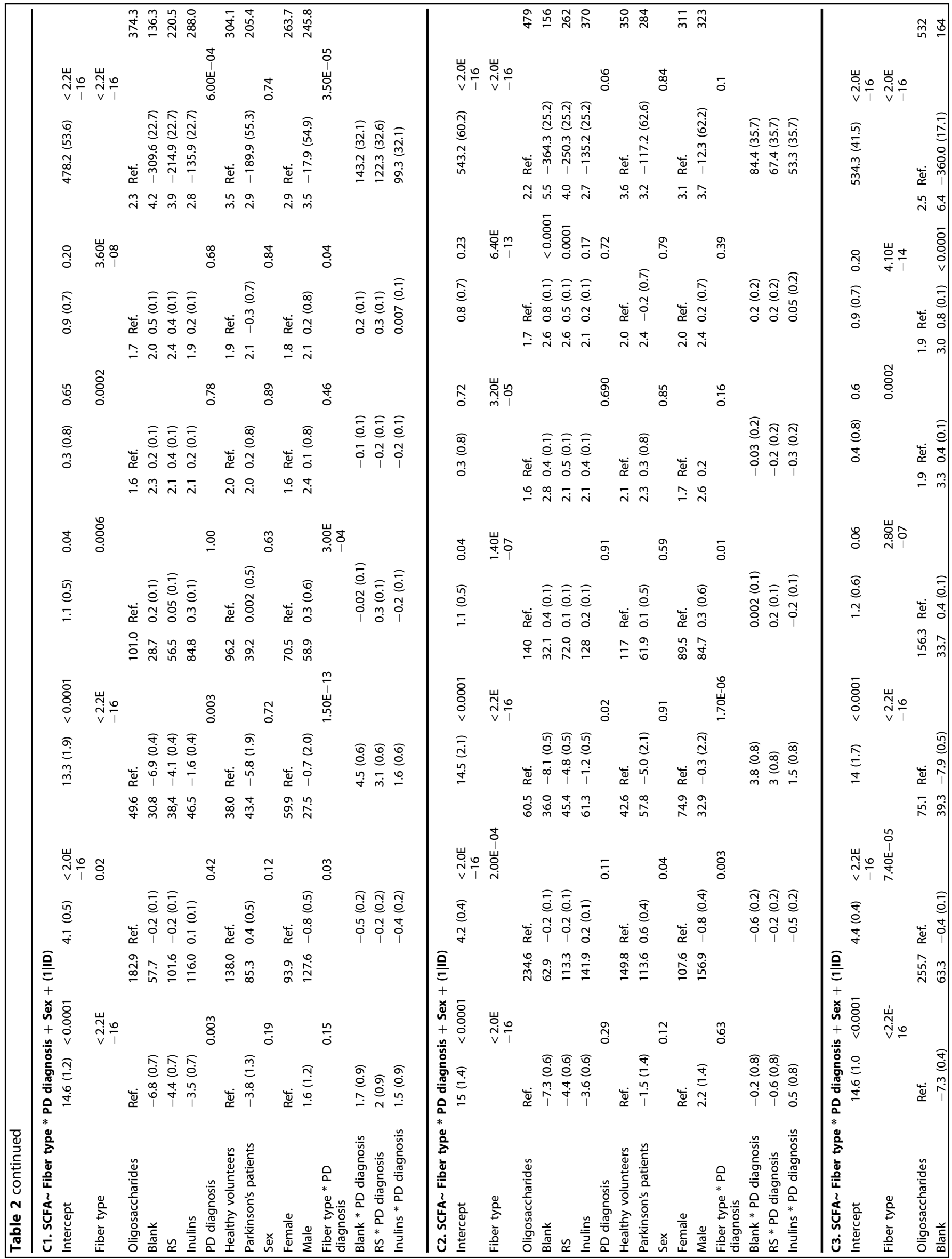




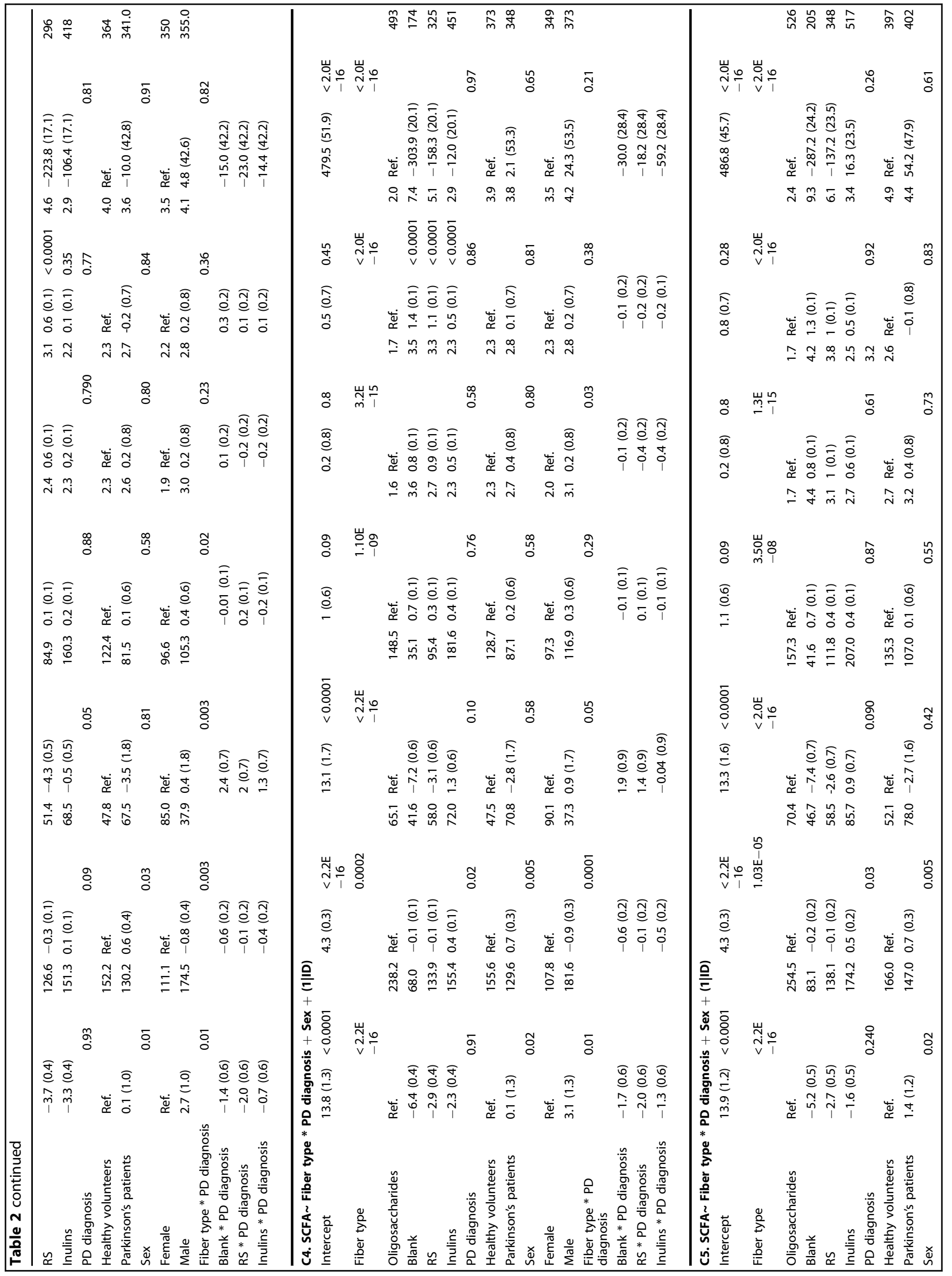




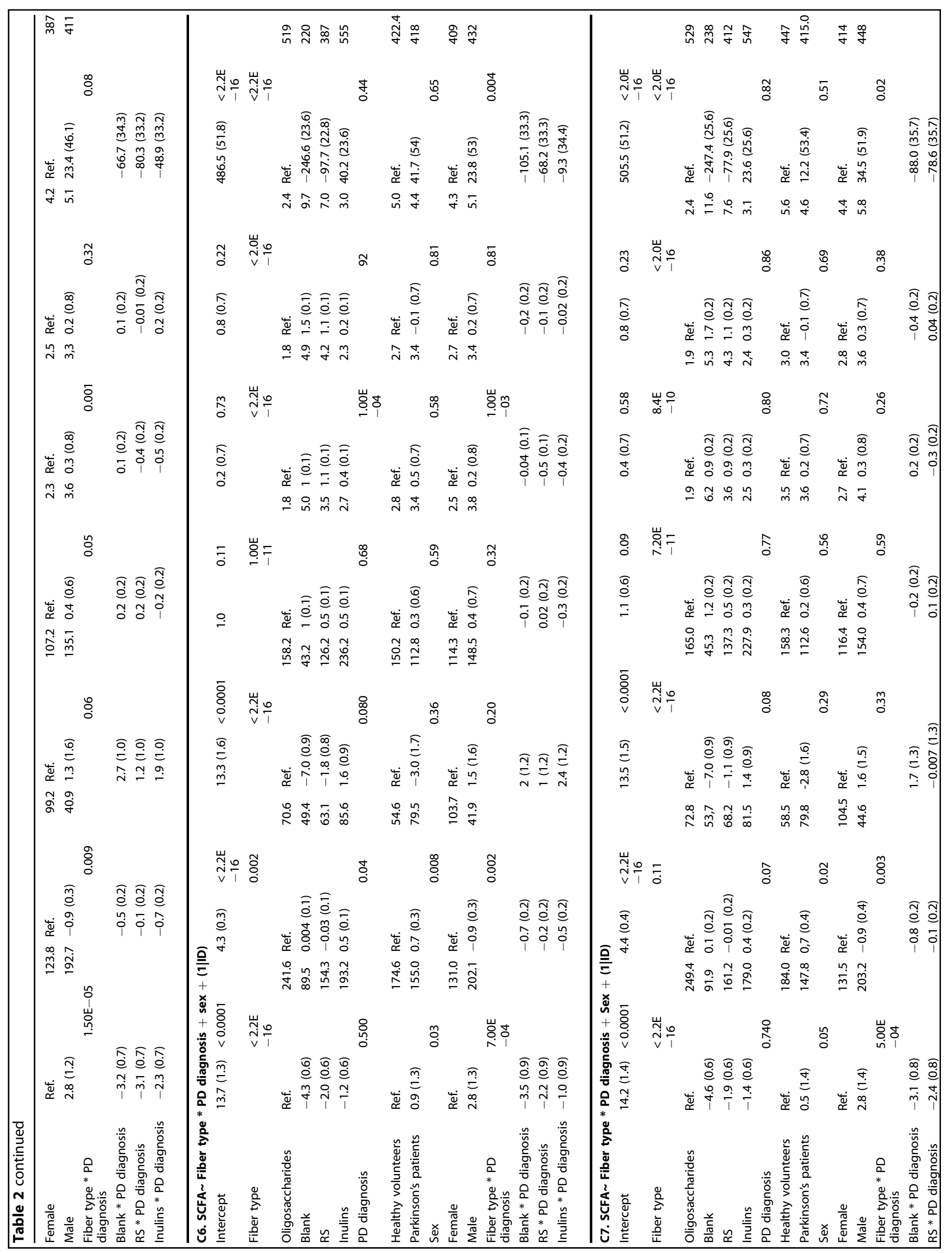




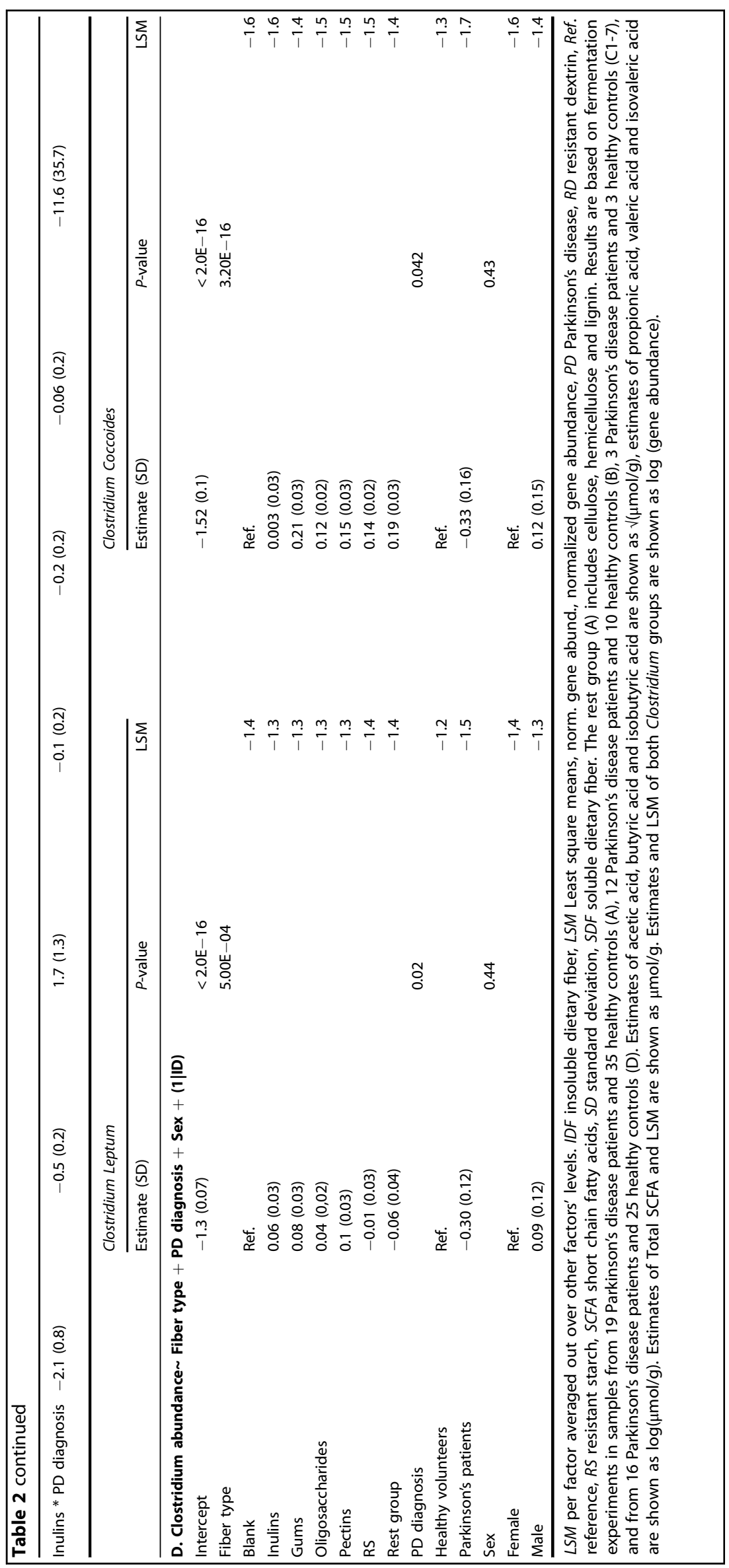


Mean butyrate production after fermentation without and with different fiber types in all participants

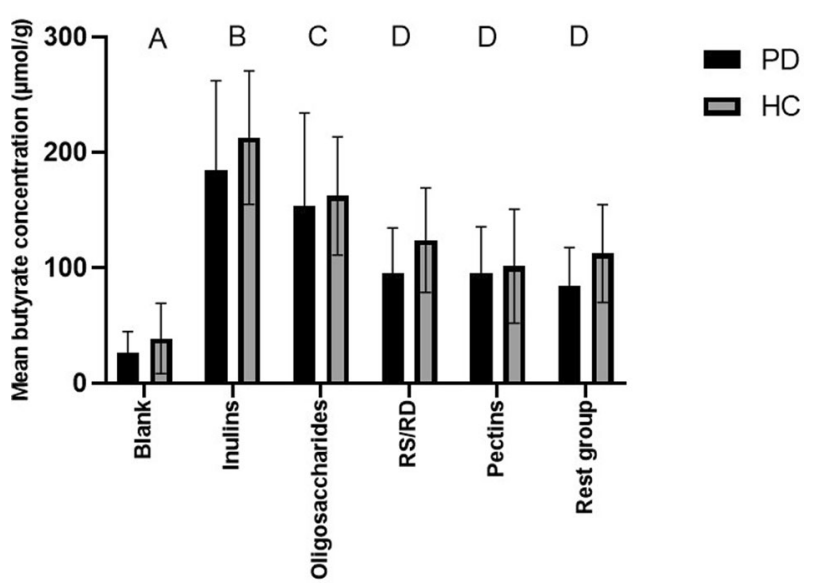

Fig. 2 Mean butyrate concentration after 24h fermentation with different types of fiber supplements. Results presented as mean \pm $\mathrm{SD}$; different letters indicate significant differences in butyrate production between fiber types; RS resistant starch, RD resistant dextrin, oligosaccharides are fructo-, galacto- and human milk oligosaccharides; rest group comprises fibers consisting of hemicelluloses, cellulose, and lignin. Differences between fiber types were post-hoc analyzed by pairwise comparisons, using Tukey to correct for multiple testing. All significant differences observed, had a $p$-value $<0.0001$

$\mathrm{C}_{\max }$ or $\mathrm{t}_{\max }$ were observed between PD patients and $\mathrm{HC}$, for inulin $\mathrm{C}_{\max }$ was almost the same between PD patients and $\mathrm{HC}(236.1 \pm$ $95.4 \mu \mathrm{mol} / \mathrm{g}$ vs. $236.0 \pm 19.9 \mu \mathrm{mol} / \mathrm{g}$ ) (Supplementary Table 4). No differences in $\mathrm{pH}$ were observed between PD patients and $\mathrm{HC}$ (Supplementary Table 5).

The next step was to investigate the effect of fiber fermentations on butyrate-producers. Univariate analysis showed a negative effect and a negative trend of PD on $C$. coccoides ( $p=$ $0.03)$ and $C$. leptum $(p=0.09)$ abundances respectively, when only taking post-incubation blanks into account. This may explain the lower AUC of SCFA in PD (see Fig. 3). The potential effect of PD medication, disease duration, and antidepressant intake on the abundance of butyrate-producers was analyzed within PD patients. No associations were found. Fiber type and PD diagnosis significantly influenced $C$. coccoides and leptum abundances, see Table 2D. Only pectin $(p=0.02)$ increased $C$. leptum abundance differently from blanks. Pectin resulted in higher $C$. leptum abundance compared to RS $(p=0.008)$ and fibers consisting of cellulose, hemicelluloses, and lignin $(p=0.006)$. No significant difference was found between other fiber types. All fiber types $(p<0.0001)$, except inulins, increased $C$. coccoides abundance. Results of Clostridium-group abundances in PD and HC are shown in Supplementary Fig. 9.

\section{Vegetable and quinoa fibers}

Univariate analyses of baseline SCFA concentrations prior to fermentation (Blank T0) demonstrated a trend $(p=0.07)$ towards lower acetate concentration in PD patients compared to HC. Univariate analyses of vegetable and quinoa fibers fermentation experiments demonstrated overall reduced production of acetate $(p=0.01)$ and total SCFA $(p=0.03)$ in PD compared to $\mathrm{HC}$, whereas a trend $(p=0.08)$ of lower butyrate production was found. Further univariate analyses are shown in Supplementary Table 2. These experiments demonstrated no associations between PD medication or antidepressant intake and SCFA production within PD patients. A trend $(p=0.06)$ towards a
Butyrate Kinetics in PD

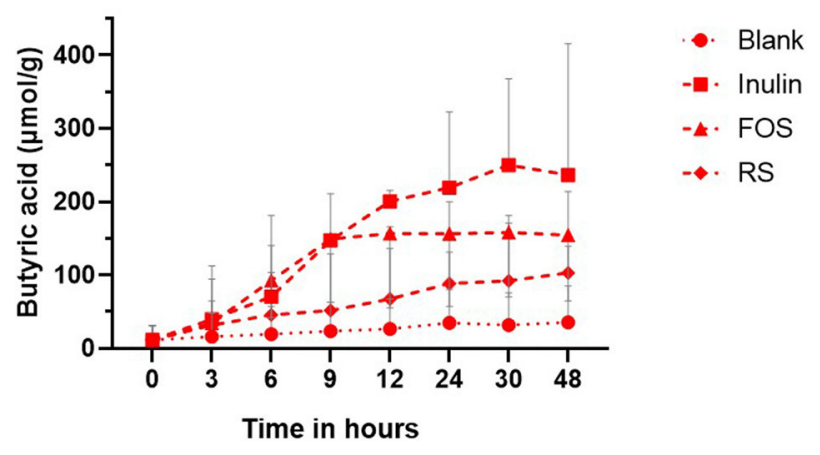

Butyrate Kinetics in $\mathrm{HC}$

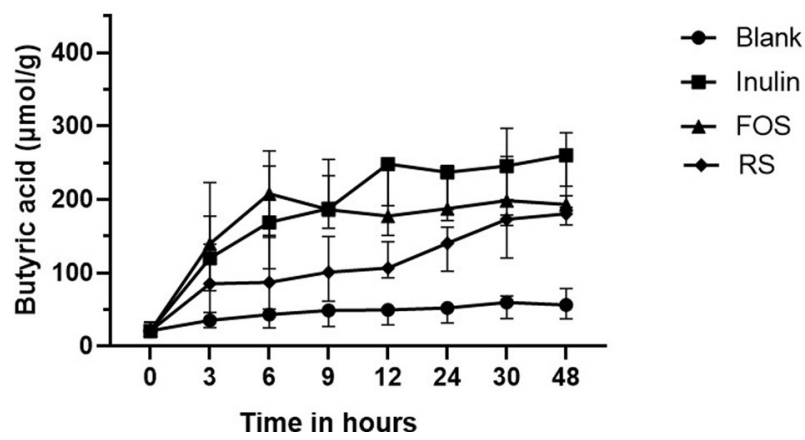

Fig. 3 Kinetics of butyrate production during $48 \mathrm{~h}$ fermentation of Orafti HP, Actilight P950 and Novelose $\mathbf{3 3 0}$ in Parkinson's disease patients and healthy controls. Results are shown as median \pm range, PD Parkinson's disease patients, $\mathrm{HC}$ healthy controls, Orafti HP is inulin; Actilight P950 is FOS; Novelose 330 is resistant starch. Butyrate kinetics were analyzed using linear mixed models per time point, sex, fiber type, PD diagnosis and interaction effect of fiber type, and PD diagnosis were added as fixed factors, the participant was added as a random factor. The kinetic profile indicated that 3-12 $\mathrm{h}$ after inulin, RS, and FOS fermentation, PD had lower butyrate production compared to $\mathrm{HC}$.

positive association between disease duration and isovalerate was found.

Multivariate models of vegetable and quinoa fermentation experiments also demonstrated PD diagnosis and fiber type to be the strongest predictors of SCFA production, see Table 2B. Soluble dietary fiber (SDF) stimulated butyrate production more $(p<$ 0.0001 ) compared to insoluble dietary fiber (IDF) (see Fig. 1). Not only inulin-rich SDF substrates increased butyrate production greatly, but also oyster mushroom stem SDF, rich in $\beta$-glucans, stimulated butyrate production considerably in PD and HC, see Supplementary Table 6 and Supplementary Fig. 10. Overall acetate production was significantly reduced in PD, while fiber stimulated acetate production. Fiber stimulated total SCFA production, whereas a trend of overall reduced production in PD was found. Multivariate analysis demonstrated no significant effect of PD on butyrate production in these experiments.

\section{DISCUSSION}

According to the authors' knowledge, this is the first study to assess dietary fiber's effect on fecal SCFA production of PD patients. Our results demonstrated that all fiber types stimulated SCFA production, in both PD and HC. However, PD diagnosis limited SCFA production and negatively influenced both Clostridium-group abundances. We only report an overall significant difference in SCFA production between PD patients and HC, and 
not a significant difference in SCFA production between PD patients and $\mathrm{HC}$ per fiber type. Interaction effects between PD and fiber type were not considered because no interaction was observed after visualization, furthermore, the consequent multiple post-hoc analyses would increase bias because of multiple testing.

Large inter-individual variability in SCFA production was observed in both groups. High inter-individual variability in gut microbiota responses to dietary interventions has been reported ${ }^{31-33}$. Studies suggest that dietary habits and baseline gut microbiome compositions influence inter-individual variability ${ }^{32}$. Participants' dietary habits were not evaluated in our study, only dietary fiber intake of the day prior to sampling. Therefore, we cannot conclude if diet or other factors are the main cause of the inter-individual variation.

Still, both fermentation experiments found similar results regarding the limited SCFA production capacity of PD patients compared to HC. Unger et al. (2016) found reduced fecal acetate, propionate, and butyrate concentrations in PD patients, compared to age-matched controls ${ }^{5}$. In contrast, our results demonstrated no influence of PD on propionate production. Our results however include the influence of fiber fermentation, whereas Unger et al. (2016) only investigated basal SCFA concentrations ${ }^{5}$. Our results demonstrated a significant lower basal acetate concentration and a trend towards lower butyrate concentration in the PD patients compared to $\mathrm{HC}$ included in the fiber supplement experiments. In the vegetable and quinoa fibers experiments, a trend towards lower basal acetate concentration in PD patients compared to HC was found. These discrepancies in our results and those of Unger et al. (2016) might be explained by the lower number of participants in our study or potential differences in dietary intake.

A positive association between COMT inhibitors and total SCFA was found in the fiber supplement, but not in vegetable and quinoa fiber experiments. In the latter experiments however, a trend $(p=0.05)$ towards a positive association between COMT inhibitors and butyrate production was found. This is in contrast with findings of Unger et al. $(2016)^{5}$ and a recent pilot study by Grün et al. (2020), that found a negative association between entacapone, but not other COMT inhibitors, and Faecalibacterium prausnitzii (a butyrate-producer) and a trend towards reduced fecal butyrate ${ }^{34}$. It is not clear how COMT inhibitors are associated with SCFA production. Studies by Unger et al. (2016) and Grün et al. (2020) are based on relatively small sample size and also in our study only 7 PD patients reported COMT inhibitor intake. Another discrepancy between our study and the studies by Unger et al. (2016) and Grün et al. (2020) is that in our study SCFA production was stimulated in vitro, whereas both Unger et al. (2016) and Grün et al. (2020) investigated the basal butyrate concentration in fecal samples. The latter refers to butyrate that is not absorbed by the colonocytes in vivo and is consequently excreted. In vivo, COMT inhibitors may be associated with higher SCFA production, which in result may also lead to increased absorption. Further studies are needed to elucidate the potential association between COMT inhibitors and SCFA production.

A negative association and negative trend of levodopa between isobutyrate and isovalerate production were observed in our study. Isobutyrate and isovalerate are protein fermentation end products ${ }^{35}$. Due to food-drug interaction between dietary amino acids and levodopa, PD patients are advised not to combine levodopa and protein intake ${ }^{36}$. This may explain the observed association.

Inulins had the largest effect on SCFA production in both PD and $\mathrm{HC}$, which is consistent with literature ${ }^{37,38}$. Koecher et al. (2014) reported similar mean total SCFA production after $24 \mathrm{~h}$ inulin fermentation in HC aged 24-32 years to our results (643 \pm $59 \mathrm{mmol} / \mathrm{l}$ versus our $560.3 \pm 17.7 \mu \mathrm{mol} / \mathrm{g}$ in $\mathrm{PD}$ and $592.5 \pm$ $21.9 \mu \mathrm{mol} / \mathrm{g}$ in age-matched $\mathrm{HC}$, reported as mean $\pm \mathrm{SEM}$ ). Although RS is currently considered the most butyrogenic substrate $^{25,39}$, our results indicated a lesser effect of RS compared to inulins, confirming previous studies ${ }^{38,40}$. This may be due to RS' lower fermentation rate ${ }^{25}$, as also indicated by our kinetics experiment.

PD diagnosis negatively influenced relative $C$. coccoides and $C$. leptum abundances, consistent with reported lower abundances of butyrate-producers ${ }^{2-5,7,41}$. This is probably part of the mechanism behind PD diagnosis' limiting effect on SCFA production. In contrast to our results, Qian et al. (2018) found an increase in C. leptum abundance in PD patients ${ }^{42}$. No information regarding dietary fiber intake was provided, however differences in fiber intake may explain this inconsistency. Our study clearly shows that fiber supplements significantly influenced Clostridium-group abundances in vitro, which is consistent with other studies ${ }^{43-45}$. Though inulins resulted in the largest butyrate increase, inulins were not associated with either Clostridium-group abundances. This suggests that increased butyrate production following inulin fermentation may be an effect of cross-feeding ${ }^{46}$.

Kinetics showed fiber fermentation resulted in lower butyrate production in PD compared to $\mathrm{HC}$ during the first $12 \mathrm{~h}$, potentially due to reduced butyrate-producers. At $48 \mathrm{~h}$ butyrate production of PD was similar to $\mathrm{HC}$, indicating that PD patients' remaining butyrate-producers still ferment fiber, but with a slower production start. Remarkably, $C_{\max }$ of inulin fermentation was similar in PD and HC, whereas the AUC of all fibers was higher in HC. The effect of PD diagnosis on AUC was not statistically significant, probably because of the high variability in SCFA production and the limited sample size of the kinetics experiments. Increased daily fiber intake (depending on fiber type) may activate butyrateproducers in PD patients, thereby increasing the fermentation rate. This could not be confirmed by dietary fiber intake, however, those results are based on one day and may not accurately represent usual fiber intake. Brahma et al. (2017) demonstrated that gut microbiota from people on a high-quality diet (characterized by high fiber intake) were more equipped for butyrate production compared to those of people with a lower quality diet ${ }^{47}$. Longer reporting periods of dietary fiber intake and the association with butyrate production in PD patients would be interesting to investigate further.

Vegetable-derived SDF increased butyrate production more than IDF in both PD and HC, which could be explained by solubility or by fiber composition ${ }^{48,49}$. Belgian endive roots, chicory roots, and black salsify are inulin-rich ${ }^{50-52}$, which also demonstrated butyrogenic effects in PD and $\mathrm{HC}$ in fiber supplement experiments. Oyster mushrooms are rich in $\beta$-glucans ${ }^{53}$, hemicellulose that has bifidogenic properties and influences SCFA production ${ }^{54}$. Consistent with our results, fungal $\beta$-glucans are promising prebiotic candidates, which were shown to increase Faecalibacterium prausnitzii and butyrate in fecal samples of $\mathrm{HC}$ older than 65 years ${ }^{55}$. Vegetable and quinoa IDF fractions are rich in hemicelluloses, cellulose, and lignin ${ }^{56-61}$. Cellulose is generally not completely fermented in the gut, because of its structure ${ }^{49}$, potentially explaining the lower butyrate production of IDF.

The current study has some limitations. A static fermentation model was used, which is effective for the fermentation extent/ rate assessment of dietary fibers and their SCFA production, however it is limited since it does not consider SCFA absorption. Recruitment of PD patients was difficult, participants often did not meet the in- or exclusion criteria or were unwilling to participate, and the amount of fecal sample collected by participants was often (too) small, thereby limiting the number of fibers to be tested and the number of analyses that could be carried out. The ratio of men to women were significantly different between PD patients and HC. Although sex was included as a potential confounding variable in the linear mixed models, this still may have impacted our results. The use of steroids was not used as an exclusion criterion, although steroid use has been reported to impact the gut microbiome. However, only $1 \mathrm{HC}$ reported the use 
of steroids, therefore the impact on our results should be limited. Though the kinetics results provide an interesting first look into PD patients' butyrate production, they are based upon limited sample size, thereby limiting external validity. Nonetheless, this study provides a useful first insight into dietary fiber's effect on SCFA production in PD patients. Current findings, however, do not allow to provide any dose-response relationship beneficial for PD patients. In future studies, it would be interesting to validate these findings in colonic mucosal samples and to use a more comprehensive approach to gut microbiota characterization through the use of next-generation sequencing, proteomics, and pathway analysis. Furthermore, reporting results by specific PD phenotypes may have added value, as it has been reported that the gut microbiome differs between different clinical PD phenotypes ${ }^{7}$.

To conclude, this study demonstrates that dietary fiber stimulates butyrate production in PD patients despite decreased butyrate-producing bacteria. However, the butyrate production remains reduced compared to $\mathrm{HC}$. Inulins in contrast to RS increase butyrate production most in both PD and $\mathrm{HC}$, however, the SCFA production start is indicated to be slower in PD compared to $\mathrm{HC}$. Of the vegetables, both the inulin-rich vegetables as the $\beta$-glucan-rich oyster mushrooms demonstrated butyrogenic effects. Dietary fiber intake may be a promising approach in PD, but further in vivo research is needed to investigate increased fiber intake's effect on plasma SCFA levels and motor symptoms.

\section{METHODS}

\section{Study design}

An in vitro study was conducted between November 2018 and November 2019 , in fecal samples of PD patients and HC of similar age. The study consisted of fecal sample collection for fermentation experiments, a questionnaire about general health (including weight loss evaluation, based on NRS 2002 methodology ${ }^{62}$ ) and medication intake and a fiber intake screening questionnaire based on the day before sample collection $^{63}$. In accordance with the advice of the Ethics Committee of the University of Leuven, participant data was anonymized, therefore no written informed consent was obtained. However, all participants provided oral informed consent prior to participating in the study. The study protocol complied with the Helsinki declaration and was approved by the Ethics Committee of the University of Leuven $\left(11^{\text {th }}\right.$ of October 2018-Reference B322201837674 - S61782). To evaluate SCFA production in PD, a stepwise approach was used. First fermentation experiments were performed using fiber supplements, second SCFA production kinetics during fermentation with three fiber types was examined and third fibers' effects on two butyrate-producing bacterial groups were assessed. Finally, to evaluate vegetables' potential, fermentation experiments were performed with vegetable-derived fibers.

\section{Study population}

$\mathrm{PD}$ patients and $\mathrm{HC}$ (men and postmenopausal women) were recruited in collaboration with a regional hospital (AZ Sint-Jan - Bruges) and regional departments of the patient's organization 'Flemish Parkinson Association'. Inclusion criteria were age between 55 and 70 and BMl between $18.5-25 \mathrm{~kg} / \mathrm{m}^{2}$, for PD patients idiopathic Parkinson's disease diagnosis was added. Exclusion criteria were antibiotics use or use of pre- and probiotics for 3 months prior to the study, prior gastrointestinal surgery, diagnosis of atypical or secondary parkinsonism or gastrointestinal diseases, including Crohn's disease, colorectal cancer, and colitis ulcerosa.

\section{Pretreatment}

All fiber supplements, selected vegetables, quinoa varieties, and their sources used in the fermentation experiments are listed in Supplementary Table 7. Vegetables were acquired fresh, except kale (freshly frozen) and black salsify (blanched and cooled). They were comminuted before airdrying at $60^{\circ} \mathrm{C}$. Quinoa and dried vegetables were ground using an Ultracentrifugal Mill (Retsch, Germany) with a $750 \mu \mathrm{m}$ sieve before purification. All fiber substrates underwent purification prior to fermentation, to remove most proteins and mono- and disaccharides present, according to Dalgetty and Baik (2003), with some modifications ${ }^{64,65}$. Purification resulted in a SDF and/or IDF substrate. Substrates underwent a water-based fractionation into a soluble and insoluble fraction, when applicable. In the soluble fraction, proteins were precipitated by $\mathrm{pH}$ adjustment from $\mathrm{pH} 3$ to $\mathrm{pH}$ 9, and mono- and disaccharides were removed through nanofiltration using the Alfa Laval LabStak $^{\text {TM }}$ M20 module (Alfa Laval, Sweden) ${ }^{64-67}$. Membranes (Alfa Laval, Sweden) had a 300 Dalton molecular weight cut-off. Purified soluble fractions were stored at $-20^{\circ} \mathrm{C}$ before freeze-drying. The insoluble fraction obtained after water-based fractionation was, when present, wet-screened through sieves ranging from $56-710 \mu \mathrm{m}^{64}$. The collected sediment was treated with alpha-amylase (MATS L Classic, $>7400$ Thermostable aamylase units per gram, IMCD, The Netherlands) at $70^{\circ} \mathrm{C}$ during $30 \mathrm{~min}^{65}$. After centrifugation, the residue was collected and stored at $-20^{\circ} \mathrm{C}$ before freeze-drying. Freeze-dried powders were sterilized using gamma sterilization (dose of 15kGy) carried out by Synergy Health, the Netherlands.

To control purification, soluble carbohydrates were analyzed in fiber supplements, vegetable, and quinoa fiber substrates ${ }^{68,69}$. Only in Belgian endive roots, black salsify, chicory roots, and oyster mushroom stems, SDF was purified since most vegetables and all quinoa samples had an SDF concentration equal to or below mono- and disaccharides concentration, which complicated purification. Vegetable and quinoa substrates were analyzed regarding polyphenol ${ }^{70,71}$, protein ${ }^{72}$, and starch content (Megazyme Digestible and Resistant Starch Assay Kit, Megazyme, Ireland) and anti-oxidative capacities ${ }^{73-75}$. Results are shown in Supplementary Tables 8-10.

\section{Fermentation}

All fiber substrates used in fermentation experiments are shown in Supplementary Table 7. Each substrate was evaluated in a minimum of 8 fermentation experiments (fecal samples of min. 4 PD patients and $4 \mathrm{HC}$ ), see Supplementary Table 1. Fiber supplement fermentations were carried out in samples of $19 \mathrm{PD}$ patients and $35 \mathrm{HC}$. For vegetable and quinoa fiber fermentation, samples of 12 PD patients and $10 \mathrm{HC}$ were used, of which respectively 7 and 6 were also included in the fiber supplements experiment. Fecal samples were collected in recipients containing Oxoid AnaeroGen $3.5 \mathrm{~L}$ Sachet (Thermo Fisher Scientific, USA) and stored at $4{ }^{\circ} \mathrm{C}$. Participants were requested to report the date and time of sampling, time between sampling and last defecation and Bristol Stool Form Scale $(B S F S)^{76}$. Sample collection was based on previously published studies ${ }^{77-79}$. The use of Oxoid Anaerogen during sample collection induces an anaerobic environment (oxygen level below 1\%) in the sample recipient as soon as it is closed, which has been demonstrated to maintain the viability of $>90 \%$ of the extremely oxygen-sensitive gut microbiota ${ }^{80}$

Fecal samples were transported to the lab on ice within $24 \mathrm{~h}$ after collection. In the lab, the sample was introduced in an anaerobic cabinet (Whitley A35 Workstation, Don Whitley Scientific, UK) and homogenized with anaerobic phosphate-buffered saline (Thermo Fisher Scientific, USA) (1 in 10 dilution) into a fecal slurry. Aliquots of $5 \mathrm{ml}$ slurry were made, dietary fibers $(1 \% \mathrm{w} / \mathrm{v})$ were added and anaerobically incubated during $24 \mathrm{~h}$ at $37^{\circ} \mathrm{C}$. Slurry without fiber was incubated as a negative control.

Each incubation was done in triplicate. After incubation, samples were placed on ice to cease fermentation and stored at $-80^{\circ} \mathrm{C}$ until further analysis. In a subset of participants, slurry samples without fiber were collected before incubation and stored at $-80^{\circ} \mathrm{C}$ until analysis. These samples were used to assess baseline SCFA concentration.

Fermentation kinetics were studied using inulin, FOS, and RS in anaerobic fermentation during $48 \mathrm{~h}$, with 8 sampling points: baseline, 3 , $6,9,12,24,30$, and $48 \mathrm{~h}$. Inulin, FOS, and RS were chosen because these fibers have already been extensively studied and are known for their butyrogenic effects ${ }^{81}$. Kinetics of these 3 fibers were investigated using fecal samples of 3 PD patients and 3 HC (for both PD patients and HC, samples were collected from 2 men and 1 woman), following the abovedescribed method. These experiments were carried out to investigate if SCFA of PD patients are produced in the same velocity and quantities as in $\mathrm{HC}$.

\section{SCFA analysis}

Standards for SCFA analysis (acetic acid, acetic acid D4, butyric acid, isobutyric acid, isovaleric acid, propionic acid, valeric acid, and valeric acid D9) were purchased from Sigma Aldrich, USA. Formic acid was purchased from Biosolve, the Netherlands. 
For analysis of acetate, propionate, butyrate, valerate, isobutyrate, and isovalerate in fecal slurries, samples were prepared as follows. Of the slurry, $200 \mu \mathrm{l}$ was added to a $20 \mathrm{~mL}$ headspace vial (Research Institute for Chromatography (RIC), Belgium). Also, $2 \mathrm{~g}$ of $\mathrm{NaCl}, 100 \mu \mathrm{l}$ of internal standards (IS) acetic acid-D4 $(0.1 \mathrm{mmol} / \mathrm{ml})$, and valeric acid-D9 $(0.1 \mathrm{mmol} /$ $\mathrm{ml}$ ) were added. Deionized water containing $0.1 \%$ formic acid was added until a total volume of $10 \mathrm{ml}$ was obtained, afterwards the vial was capped. Quantification was done based on relative areas (using IS) and using external standard curves of reference analytical standards. Total SCFA was determined as the sum of all SCFA.

SCFA were extracted and analyzed using automated headspace solidphase microextraction - gas chromatography-mass spectrometry with a Gertsel MPS sampler coupled to an Agilent 8890GC and 5977B GCMSD (Agilent, USA). After sample preparation, the vial was incubated for $10 \mathrm{~min}$ at $45^{\circ} \mathrm{C}$ and agitated at $250 \mathrm{rpm}$, followed by $40 \mathrm{~min}$ extraction at the same temperature with a Supelco 50/30 $\mu \mathrm{m}$ Divinylbenzene/Carboxen/ Polydimethylsiloxane fiber (Supelco, USA). The SPME fiber was desorbed in splitless mode at $250^{\circ} \mathrm{C}$ and analytes were separated on an HP-FFAP column $(25 \mathrm{~m} \times 0.2 \mathrm{~mm} \times 0.33 \mu \mathrm{m})$ (Agilent, USA) using a helium flow rate of $1.6 \mathrm{ml} / \mathrm{min}$. Oven temperature program was set as follows: start at $60^{\circ} \mathrm{C}$, hold for $1 \mathrm{~min}$, then raised to $230^{\circ} \mathrm{C}$ at a rate of $10^{\circ} \mathrm{C} / \mathrm{min}$ and hold for $2 \mathrm{~min}$. Compounds were ionised through electron impact ionization and the mass spectrometer was operated in selected ion monitoring (SIM)/ SCAN mode. Data were processed using the acquired SIM data.

\section{Quantitative real-time PCR}

DNA was extracted from $2 \mathrm{ml}$ slurry using QIAamp Fast DNA Stool mini kit (Qiagen, Germany) according to Knudsen et al. (2016) ${ }^{82}$. Clostridium leptum and Clostridium coccoides groups, to which many butyrate-producing bacteria belong ${ }^{83}$, were quantified using GPCR in slurries after $24 \mathrm{~h}$ fermentation with and without a selection of fiber supplements, see Supplementary Table 7. For this selection, one representative per fiber type was chosen. QPCR was carried out on a Lightcycler 480 Real-time PCR system (Roche, Germany) using SYBR Green. Per fiber, slurries from 5 PD patients and $5 \mathrm{HC}$ were analyzed for the quantification of Clostridium coccoides and Clostridium leptum groups using QPCR. DNA extracts were eluated using $100 \mu \mathrm{l}$ elution buffer and DNA concentration was checked using a Quantus fluorometer (Promega Corporation, USA). Per slurry, DNA extracts of two biological replicates were analyzed. Each DNA extract was 100-fold diluted to eliminate PCR-inhibition and analyzed in triplicate. Total $16 \mathrm{~S}$ rRNA gene was quantified as a proxy for bacterial load. Previously published primers for Clostridium group-specific and eubacterial 16 S rRNA genes were BLASTed and aligned with GenBank sequences to ascertain their location ${ }^{84-87}$. Subsequently, a plasmid (containing eubacterial $16 \mathrm{~S}$ rRNA gene target) and a gBlock containing 2 consecutive sequences of interest (a 246 bp sequence of $16 \mathrm{~S}$ rRNA of Clostridium leptum (consisting of region 914-1159 bp of Genbank accession NR_114789.1) and a 440 bp sequence of $16 \mathrm{~S}$ rRNA of Dorea formicigenerans (consisting of region 466-905 of Genbank NR_044645.2)) separated by the nucleotides AT, were designed and both obtained from Integrated DNA Technologies, USA. Tenfold serial dilutions of the plasmid and gblock were used as standards for quantification. SsoAdvanced SYBR green supermix was purchased from BioRad Laboratories, USA. The reaction mixture consisted of $5 \mu$ template DNA, $12.5 \mu \mathrm{l}$ SsoAdvanced SYBR green supermix, the correct amount of each primer and sterile water to obtain a total volume of $25 \mu \mathrm{l}$. For the amplification of Clostridium group-specific 16S rRNA gene-targets, an activation step of $5 \mathrm{~min}$ at $94^{\circ} \mathrm{C}$ was followed by 30 or 32 cycli (for Clostridium coccoides and leptum, respectively) of $20 \mathrm{~s}$ at $94{ }^{\circ} \mathrm{C}, 20 \mathrm{~s}$ at $50^{\circ} \mathrm{C}$ and $15 \mathrm{~s}$ at $72^{\circ} \mathrm{C}$ and one cycle of $15 \mathrm{~s}$ at $94^{\circ} \mathrm{C}$. For the amplification of the eubacterial $16 \mathrm{~S}$ rRNA gene target, an activation step of $10 \mathrm{~min}$ at $95^{\circ} \mathrm{C}$ was followed by 30 cycles of $15 \mathrm{~s}$ at $95^{\circ} \mathrm{C}$ and $1 \mathrm{~min}$ at $60^{\circ} \mathrm{C}$. The specificity of the reaction products was assessed by melting curve analysis. This was performed by gradually increasing the temperature from 60 to $95^{\circ} \mathrm{C}$ at a rate of $0.2^{\circ} \mathrm{C} / \mathrm{s}$, with continuous fluorescence collection. Details of primer sequences and primer concentration are shown in Supplementary Table 11.

\section{Data analysis}

SCFA data was analyzed using Agilent MassHunter Quantitative Data Analysis (Agilent, USA). QPCR results were analyzed using Lightcycler 480 Software (Roche, Germany). Results of SCFA analysis were corrected for dilution. Relative abundances of Clostridium group-specific $16 \mathrm{~S}$ rRNA gene targets were calculated by dividing their abundance by total eubacterial
$16 \mathrm{~S}$ rRNA gene abundance. This normalization was carried out to account for differences in extraction efficiency and total bacterial number.

\section{Statistical analysis}

Possible differences in sex, BSFS and medication intake between PD patients and HC were analyzed using Fisher's exact test. To evaluate potential differences in BMI, fiber intake and age between PD and HC, Student T-test and Mann-Whitney U-test was used, depending on normality (evaluated by Shapiro Wilk test). Log transformation of both Clostridium-group genes, propionate, valerate and isovalerate and square root transformation of acetate, butyrate and isobutyrate was carried out to meet linear regression assumptions (normality, homoscedasticy and independence of residuals, no autocorrelation and little multicollinearity). To investigate factors that may influence Clostridium-group abundances or production of the different SCFA, linear mixed models (LMM) were used. Fiber type, PD diagnosis, age, sex, BMI, BSFS, fiber intake, sampling time, time between sampling and last defecation, weight loss, medication intake or Clostridium-groups (in SCFA models) were added as fixed factor, participant was used as random factor in univariate analyses. In final multivariate models fiber type, PD diagnosis, sex were added as fixed factors and participant as a random factor. Sex was included as potential confounding variable, due to the imbalance of men/women between PD and HC. No interaction effects were considered in the Clostridium or SCFA models. SCFA kinetics were analyzed using LMM per time point, sex, PD diagnosis, fiber type and interaction effect of fiber type and PD diagnosis were added as fixed factors, participant was added as a random factor. Residuals' normality in all models was assessed using histograms and Q-Q plots, homoscedasticy, and independence of residuals were evaluated by plotting residuals. Post-hoc analysis of blanks (ref) versus fiber types was carried out for LMM of Clostridium-groups and SCFA, using Dunnett's test to correct for multiple testing. Post-hoc pairwise comparisons between beforehand selected fiber types (inulins, oligosaccharides, pectins, RS, and the combination of hemicelluloses, cellulose, and lignin) were carried out, using the Tukey test to correct for multiple testing. The number of posthoc tests was limited, to reduce the risk of type I errors. Analyses were carried out using RStudio 1.1.456. Statistical significance was determined as $p<0.05$. Following $R$ packages were used for statistical analyses: emmeans, FSA, GGally, ggplot2, Hmisc, Imtest, Ime4, nIme, ImerTest, MuMIn, and psych.

\section{Reporting summary}

Further information on research design is available in the Nature Research Reporting Summary linked to this article.

\section{DATA AVAILABILITY}

The datasets generated and/or analyzed during the current study and the supplementary information are available in the Figshare repository, https://doi.org/ 10.6084/m9.figshare.13238045.

Received: 14 November 2020; Accepted: 9 July 2021; Published online: 13 August 2021

\section{REFERENCES}

1. Elfil, M., Kamel, S., Kandil, M., Koo, B. B. \& Schaefer, S. M. Implications of the gut microbiome in Parkinson's disease. Mov. Disord. 35, 921-933 (2020).

2. Keshavarzian, A. et al. Colonic bacterial composition in Parkinson's disease. Mov. Disord.: Off. J. Mov. Disord. Soc. 30, 1351-1360 (2015).

3. Hill-Burns, E. M. et al. Parkinson's disease and Parkinson's disease medications have distinct signatures of the gut microbiome. Mov. Disord.: Off. J. Mov. Disord. Soc. 32, 739-749 (2017).

4. Petrov, V. A. et al. Analysis of Gut Microbiota in Patients with Parkinson's Disease. Bull. Exp. Biol. Med. 162, 734-737 (2017).

5. Unger, M. M. et al. Short chain fatty acids and gut microbiota differ between patients with Parkinson's disease and age-matched controls. Parkinsonism Relat. Disord. 32, 66-72 (2016).

6. Li, W. et al. Structural changes of gut microbiota in Parkinson's disease and its correlation with clinical features. Science China. Life sciences. https://doi.org/ 10.1007/s11427-016-9001-4 (2017). 
7. Scheperjans, F. et al. Gut microbiota are related to Parkinson's disease and clinical phenotype. Mov. Disord.: Off. J. Mov. Disord. Soc. 30, 350-358 (2015).

8. Dalile, B., Van Oudenhove, L., Vervliet, B. \& Verbeke, K. The role of short-chain fatty acids in microbiota-gut-brain communication. Nat. Rev. Gastroenterol. Hepatol. 16, 461-478 (2019).

9. Kekuda, R., Manoharan, P., Baseler, W. \& Sundaram, U. Monocarboxylate 4 mediated butyrate transport in a rat intestinal epithelial cell line. Digestive Dis. Sci. 58, 660-667 (2013)

10. Bachmann, C., Colombo, J.-P. \& Berüter, J. Short chain fatty acids in plasma and brain: quantitative determination by gas chromatography. Clin. Chim. Acta. 92, 153-159 (1979)

11. St Laurent, R., O'Brien, L. M. \& Ahmad, S. T. Sodium butyrate improves locomotor impairment and early mortality in a rotenone-induced Drosophila model of Parkinson's disease. Neuroscience 246, 382-390 (2013).

12. Paiva, I. et al. Sodium butyrate rescues dopaminergic cells from alpha-synuclein induced transcriptional deregulation and DNA damage. Hum. Mol. Genet. 26, 2231-2246 (2017).

13. Sharma, S., Taliyan, R. \& Singh, S. Beneficial effects of sodium butyrate in 6-OHDA induced neurotoxicity and behavioral abnormalities: Modulation of histone deacetylase activity. Behavioural brain Res. 291, 306-314 (2015).

14. Matt, S. M. et al. Butyrate and dietary soluble fiber improve neuroinflammation associated with aging in mice. Front. Immunol. 9, https://doi.org/10.3389/ fimmu.2018.01832 (2018)

15. Sampson, T. R. et al. Gut microbiota regulate motor deficits and neuroinflammation in a model of Parkinson's disease. Cell 167, 1469-1480 (2016). e1412.

16. Mulak, A. A controversy on the role of short-chain fatty acids in the pathogenesis of Parkinson's disease. Movement disorders: official journal of the Movement Disorder Society. https://doi.org/10.1002/mds.27304 (2018).

17. Kratsman, N., Getselter, D. \& Elliott, E. Sodium butyrate attenuates social behavior deficits and modifies the transcription of inhibitory/excitatory genes in the frontal cortex of an autism model. Neuropharmacology 102, 136-145 (2016).

18. Hamer, H. M. et al. Effect of butyrate enemas on inflammation and antioxidant status in the colonic mucosa of patients with ulcerative colitis in remission. Clin. Nutr. 29, 738-744 (2010).

19. Facchin, S. et al. Microbiota changes induced by microencapsulated sodium butyrate in patients with inflammatory bowel disease. Neurogastroenterology \& Motility n/a, e13914, https://doi.org/10.1111/nmo.13914 (2020).

20. Chen, R. et al. Transplantation of fecal microbiota rich in short chain fatty acids and butyric acid treat cerebral ischemic stroke by regulating gut microbiota. Pharmacol. Res. 148, 104403 (2019)

21. Sun, M.-F. et al. Neuroprotective effects of fecal microbiota transplantation on MPTP-induced Parkinson's disease mice: Gut microbiota, glial reaction and TLR4/ TNF-a signaling pathway. Brain, behavior, and immunity. https://doi.org/10.1016/j. bbi.2018.02.005 (2018).

22. Markowiak-Kopeć, P. \& Śliżewska, K. The effect of probiotics on the production of short-chain fatty acids by human intestinal microbiome. Nutrients 12, 1107 (2020).

23. Hsieh, T.-H. et al. Probiotics alleviate the progressive deterioration of motor functions in a mouse model of Parkinson's disease. Brain Sci. 10, 206 (2020).

24. Roberfroid, M. B. Inulin-type fructans: functional food ingredients. J. Nutr. 137, 2493S-2502S (2007).

25. Brouns, F., Kettlitz, B. \& Arrigoni, E. Resistant starch and "the butyrate revolution". Trends Food Sci. Technol. 13, 251-261 (2002)

26. Homayoni Rad, A., Akbarzadeh, F. \& Mehrabany, E. V. Which are more important: prebiotics or probiotics? Nutrition 28, 1196-1197 (2012).

27. Marshall, V. M. Prebiotics: development and application. Int. J. Dairy Technol. 61, 310-311 (2008)

28. Agnieszka, M. et al. Protein intake in parkinsonian patients using the EPIC food frequency questionnaire. Mov. Disord. 21, 1229-1231 (2006).

29. Barichella, M. et al. Dietary habits and neurological features of Parkinson's disease patients: Implications for practice. Clin. Nutr. 36, 1054-1061 (2017).

30. Baert, F. et al. Dietary intake of parkinson's disease patients. Front. Nutr. 7, 105 (2020).

31. Flint, H. J. The impact of nutrition on the human microbiome. Nutr. Rev. 70 S10-S13 (2012)

32. Healey, G. R., Murphy, R., Brough, L., Butts, C. A. \& Coad, J. Interindividual variability in gut microbiota and host response to dietary interventions. Nutr. Rev. 75, 1059-1080 (2017).

33. Hughes, R. L., Kable, M. E., Marco, M. \& Keim, N. L. The Role of the gut microbiome in predicting response to diet and the development of precision nutrition models. part ii: results. Adv. Nutr. 10, 979-998 (2019).

34. Grün, D. et al. Impact of oral COMT-inhibitors on gut microbiota and short chain fatty acids in Parkinson's disease. Parkinsonism Relat. Disord. 70, 20-22 (2020).

35. Cardona, M. E. et al. Correlation between faecal iso-butyric and iso-valeric acids in different species. Microb. Ecol. Health Dis. 17, 177-182 (2005).
36. Guebila, M. B. \& Thiele, I. Model-based dietary optimization for late-stage, levodopa-treated, Parkinson's disease patients. npj Syst. Biol. Appl. 2, 16013 (2016).

37. Koecher, K. J. et al. Estimation and interpretation of fermentation in the gut coupling results from a $24 \mathrm{~h}$ batch in vitro system with fecal measurements from a human intervention feeding study using fructo-oligosaccharides, inulin, gum acacia, and pea fiber. J. Agric. food Chem. 62, 1332-1337 (2014).

38. Yang, J., Martínez, I., Walter, J., Keshavarzian, A. \& Rose, D. J. In vitro characterization of the impact of selected dietary fibers on fecal microbiota composition and short chain fatty acid production. Anaerobe 23, 74-81 (2013).

39. Birt, D. F. et al. Resistant starch: promise for improving human health. Adv. Nutr. 4 587-601 (2013).

40. Rahat-Rozenbloom, S., Fernandes, J., Cheng, J., Gloor, G. B. \& Wolever, T. M. S. The acute effects of inulin and resistant starch on postprandial serum short-chain fatty acids and second-meal glycemic response in lean and overweight humans. Eur. J. Clin. Nutr. 71, 227-233 (2017)

41. Li, F. et al. Alteration of the fecal microbiota in north-eastern Han Chinese population with sporadic Parkinson's disease. Neurosci. Lett. https://doi.org/ 10.1016/j.neulet.2019.134297 (2019).

42. Qian, Y. et al. Alteration of the fecal microbiota in Chinese patients with Parkinson's disease. Brain, behavior, and immunity. https://doi.org/10.1016/j. bbi.2018.02.016 (2018).

43. Cantu-Jungles, T. M. et al. In vitro fermentation of Cookeina speciosa glucans stimulates the growth of the butyrogenic Clostridium cluster XIVa in a targeted way. Carbohydr. Polym. 183, 219-229 (2018).

44. Duncan, S. H. et al. Reduced dietary intake of carbohydrates by obese subjects results in decreased concentrations of butyrate and butyrate-producing bacteria in feces. Appl. Environ. Microbiol. 73, 1073-1078 (2007).

45. Chung, W. S. F. et al. Prebiotic potential of pectin and pectic oligosaccharides to promote anti-inflammatory commensal bacteria in the human colon. FEMS Microbio. Ecol. https://doi.org/10.1093/femsec/fix127 (2017).

46. Moens, F., Weckx, S. \& De Vuyst, L. Bifidobacterial inulin-type fructan degradation capacity determines cross-feeding interactions between bifidobacteria and Faecalibacterium prausnitzii. Int. J. food Microbiol. 231, 76-85 (2016).

47. Brahma, S. et al. Impact of dietary pattern of the fecal donor on in vitro fermentation properties of whole grains and brans. J. Funct. Foods 29, 281-289 (2017).

48. Slavin, J. Fiber and prebiotics: mechanisms and health benefits. Nutrients $\mathbf{5}$, 1417-1435 (2013).

49. Hamaker, B. R. \& Tuncil, Y. E. A perspective on the complexity of dietary fiber structures and their potential effect on the gut microbiota. J. Mol. Biol. 426, 3838-3850 (2014).

50. Wilson, R., Smith, J. \& Yonts, C. Chicory root yield and carbohydrate composition is influenced by cultivar selection, planting, and harvest date. Crop Sci. 44 748-752 (2004)

51. Petkova, N. Characterization of inulin from black salsify (Scorzonera hispanica I.) for food and pharmaceutical purposes. Asian J. Pharm. Clin. Res. 11, 221-225 (2018).

52. Guo, X. et al. Sequential extraction and physicochemical characterization of polysaccharides from chicory (Cichorium intybus) root pulp. Food Hydrocoll. 77, 277-285 (2018).

53. Golak-Siwulska, I., Kałużewicz, A., Spiżewski, T., Siwulski, M. \& Sobieralski, K Bioactive compounds and medicinal properties of Oyster mushrooms (Pleurotus sp.). Folia Horticulturae 30, 191 (2018).

54. Jayachandran, M., Chen, J., Chung, S. S. M. \& Xu, B. A critical review on the impacts of $\beta$-glucans on gut microbiota and human health. J. Nutritional Biochem. 61, 101-110 (2018)

55. Mitsou, E. K. et al. Effects of rich in $\beta$-glucans edible mushrooms on aging gut microbiota characteristics: an in vitro study. Molecules 25, 2806 (2020).

56. Schäfer, J., Stanojlovic, L., Trierweiler, B. \& Bunzel, M. Storage related changes of cell wall based dietary fiber components of broccoli (Brassica oleracea var. italica) stems. Food Res. Int. 93, 43-51 (2017).

57. Bourquin, L. D., Titgemeyer, E. C. \& Fahey, G. C. Jr Vegetable fiber fermentation by human fecal bacteria: cell wall polysaccharide disappearance and short-chain fatty acid production during in vitro fermentation and water-holding capacity of unfermented residues. J. Nutr. 123, 860-869 (1993).

58. Elkner, K. \& Kosson, R. Dietary fibre content and its fractional composition in cabbage as affected by cultivar earliness and sauerkraut storage period. Veg. Crops Res. Bull. 69, 165-175 (2008).

59. do Nascimento, G. E., lacomini, M. \& Cordeiro, L. M. C. New findings on green sweet pepper (Capsicum annum) pectins: Rhamnogalacturonan and type I and II arabinogalactans. Carbohydr. Polym. 171, 292-299 (2017).

60. Lamothe, L. M., Srichuwong, S., Reuhs, B. L. \& Hamaker, B. R. Quinoa (Chenopodium quinoa W.) and amaranth (Amaranthus caudatus L.) provide dietary fibres high in pectic substances and xyloglucans. Food Chem. 167, 490-496 (2015). 
61. de Evan, T., Vintimilla, A., Marcos, C. N., Ranilla, M. J. \& Carro, M. D. Evaluation of Brassica Vegetables as Potential Feed for Ruminants. Animals: an Open Access Journal from MDPI 9, https://doi.org/10.3390/ani9090588 (2019).

62. Kondrup, J., Rasmussen, H. H., Hamberg, O. \& Stanga, Z. Nutritional risk screening (NRS 2002): a new method based on an analysis of controlled clinical trials. Clin. Nutr. 22, 321-336 (2003).

63. Maes, L., Vanhauwaert, E., Hublet, L., \& Peter, V. Development, validity and reliability of a simple and quick assessment instrument for dietary fat en fibre intake for use in nutrition education. Congrès de Nutrition et Santé, Brussels (2000).

64. Dalgetty, D. D. \& Baik, B. Isolation and characterization of cotyledon fibers from peas, lentils, and chickpeas. Cereal Chem. 80, 310-315 (2003).

65. Maphosa, Y. \& Jideani, V. Physicochemical characteristics of Bambara groundnut dietary fibres extracted using wet milling. South Afr. J. Sci. 112, 1-8 (2016).

66. Pruksasri, S., Nguyen, T.-H., Haltrich, D. \& Novalin, S. Fractionation of a galactooligosaccharides solution at low and high temperature using nanofiltration. Sep. Purif. Technol. 151, 124-130 (2015).

67. Kuhn, R. C. et al. Mass transfer and transport during purification of fructooligosaccharides by nanofiltration. J. Membr. Sci. 365, 356-365 (2010).

68. Muir, J. G. et al. Measurement of short-chain carbohydrates in common Australian vegetables and fruits by high-performance liquid chromatography (HPLC). J. Agric. Food Chem. 57, 554-565 (2009).

69. Vendrell-Pascuas, S., Castellote-BargallГi, A. I. \& LГipez-Sabater, M. C. Determination of inulin in meat products by high-performance liquid chromatography with refractive index detection. J. Chromatogr. A 881, 591-597 (2000).

70. De Paepe, D. et al. An improved mass spectrometric method for identification and quantification of phenolic compounds in apple fruits. Food Chem. 136, 368-375 (2013).

71. Kips, L. et al. A novel spiral-filter press for tomato processing: process impact on phenolic compounds, carotenoids, and ascorbic acid content. J. Food Eng. 213, 27-37 (2017).

72. Saez-Plaza, P., Navas, M. J., Wybraniec, S., Michalowski, T. \& Asuero, A. G. An overview of the kjeldahl method of nitrogen determination. part ii. sample preparation, working scale, instrumental finish, and quality control. Crit. Rev. Anal. Chem. 43, 224-272 (2013).

73. Prior, R. et al. Assays for hydrophilic and lipophilic antioxidant capacity (oxygen radical absorbance capacity (ORAC(FL))) of plasma and other biological and food samples. J. Agric. Food Chem. 51, 3273-3279 (2003).

74. Franka, G. \& Dell, E. J. ORAC assay on the FLUOstar OPTIMA to determine antioxidant capacity. https://doi.org/10.1038/an2774 (2007).

75. Bernaert, N., De Clercq, H., Van Bockstaele, E., De Loose, M. \& Van Droogenbroeck, B. Antioxidant changes during postharvest processing and storage of leek (Allium ampeloprasum var. porrum). Postharvest Biol. Technol. 86, 8-16 (2013).

76. Lewis, S. J. \& Heaton, K. W. Stool form scale as a useful guide to intestinal transit time. Scand. J. Gastroenterol. 32, 920-924 (1997).

77. Kaur, A., Rose, D. J., Rumpagaporn, P., Patterson, J. A. \& Hamaker, B. R. In vitro batch fecal fermentation comparison of gas and short-chain fatty acid production using "slowly fermentable" dietary fibers. J. Food Sci. 76, H137-H142 (2011).

78. De Preter, V. et al. The prebiotic, oligofructose-enriched inulin modulates the faecal metabolite profile: an in vitro analysis. Mol. Nutr. Food Res. 54, 1791-1801 (2010).

79. Mao, Y.-H. et al. A high-molecular weight exopolysaccharide from the Cs-HK1 fungus: ultrasonic degradation, characterization and in vitro fecal fermentation. Carbohydr. Polym. 246, 116636 (2020).

80. Martínez, N., Hidalgo-Cantabrana, C., Delgado, S., Margolles, A. \& Sánchez, B. Filling the gap between collection, transport and storage of the human gut microbiota. Sci. Rep. 9, 8327-8327 (2019).

81. Riviere, A., Selak, M., Lantin, D., Leroy, F. \& De Vuyst, L. Bifidobacteria and butyrate-producing colon bacteria: importance and strategies for their stimulation in the human gut. Front. Microbiol. 7, 979 (2016).

82. Knudsen, B. E. et al. Impact of sample type and DNA isolation procedure on genomic inference of microbiome composition. mSystems 1, e00095-00016 (2016).

83. Lopetuso, L. R., Scaldaferri, F., Petito, V. \& Gasbarrini, A. Commensal Clostridia: leading players in the maintenance of gut homeostasis. Gut Pathog. 5, 23-23 (2013).

84. Matsuki, T., Watanabe, K., Fujimoto, J., Takada, T. \& Tanaka, R. Use of $16 \mathrm{~S}$ rRNA gene-targeted group-specific primers for real-time pcr analysis of predominant bacteria in human feces. Appl. Environ. Microbiol. 70, 7220-7228 (2004).

85. Matsuki, T. et al. Development of $16 \mathrm{~S}$ rRNA-gene-targeted group-specific primers for the detection and identification of predominant bacteria in human feces. Appl. Environ. Microbiol. 68, 5445 (2002).
86. Muyzer, G., de Waal, E. C. \& Uitterlinden, A. G. Profiling of complex microbial populations by denaturing gradient gel electrophoresis analysis of polymerase chain reaction-amplified genes coding for $16 \mathrm{~S}$ rRNA. Appl. Environ. Microbiol. 59, 695 (1993).

87. Yu, Y., Lee, C., Kim, J. \& Hwang, S. Group-specific primer and probe sets to detect methanogenic communities using quantitative real-time polymerase chain reaction. Biotechnol. Bioeng. 89, 670-679 (2005).

\section{ACKNOWLEDGEMENTS}

The authors thank the respective sources of the following fiber supplements and vegetables (Supplementary Table 1), Orafti HP, Orafti GR, Orafti P95, Fibruline Instant, Fibrulin XL, Fibrulose F97, Novelose 330, Actilight P950, Acacia fiber, Fibrulin S30, Liquid Oat Bran 10 Instant, Frutafit IQ, Promitor, Psyllium husk fiber, Litesse Ultra, GOS, Grindsted Pectin AMD 922, Nutriose, Pea fiber, GENU Gum RL 200, GENU Pectin

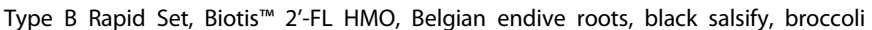
stems, cabbage, kale, white cabbage, pointed sweet bell pepper, chicory roots, Brussels sprouts, oyster mushroom stems and quinoa, who gave the fiber samples for free. This research did not receive any specific grant from funding agencies in the public, commercial, or not-for-profit sectors. However, as mentioned above, we did receive different fiber samples for free. The authors thank Eva Vanbiervliet, Doriana Ceciliani, Marie-Charlotte Gruwez, and Robbe van Bocxlaer for their assistance in carrying out the experiments. Also, thanks to Jana Szkudlarski for her help in fiber purification. A special thanks to the Flemish Parkinson Association's regional departments for their help in recruitment.

\section{AUTHOR CONTRIBUTIONS}

C.M., G.V., and F.B. developed the study design. B.B. aided in patient recruitment and provided feedback on study design, C.V.P. developed the SCFA analysis method, E.V.C. assisted in GPCR method validation and J.M. assisted in statistical analysis. F.B., C.M., and G.V. drafted the manuscript and all authors commented on and approved the final manuscript.

\section{COMPETING INTERESTS}

For all authors, no financial or other disclosures need to be made except employment at their respective institutes.

\section{ADDITIONAL INFORMATION}

Supplementary information The online version contains supplementary material available at https://doi.org/10.1038/s41531-021-00215-5.

Correspondence and requests for materials should be addressed to G.V.

Reprints and permission information is available at http://www.nature.com/ reprints

Publisher's note Springer Nature remains neutral with regard to jurisdictional claims in published maps and institutional affiliations.

Open Access This article is licensed under a Creative Commons Attribution 4.0 International License, which permits use, sharing, adaptation, distribution and reproduction in any medium or format, as long as you give appropriate credit to the original author(s) and the source, provide a link to the Creative Commons license, and indicate if changes were made. The images or other third party material in this article are included in the article's Creative Commons license, unless indicated otherwise in a credit line to the material. If material is not included in the article's Creative Commons license and your intended use is not permitted by statutory regulation or exceeds the permitted use, you will need to obtain permission directly from the copyright holder. To view a copy of this license, visit http://creativecommons. org/licenses/by/4.0/.

(c) The Author(s) 2021 